\title{
Application du calcul numérique à la dépressurisation des réservoirs cryogéniques en microgravité
}

\author{
JÉRÔME LACAPÈRE ${ }^{1, a}$ ET BRUNO VIEILle ${ }^{2}$ \\ 1 Air Liquide DTA, 38360 Sassenage, France \\ 2 CNES Direction des Lanceurs, Rond Point de l'Espace, 91000 Evry, France
}

Reçu le 14 octobre 2003, accepté le 6 avril 2004

\begin{abstract}
Résumé - Afin de contrôler la température des ergols cryogéniques dans les réservoirs des lanceurs spatiaux, il est nécessaire de maîtriser des phénomènes thermodynamiques complexes. Pour les lanceurs du futur, l'apparition de phases à microgravité augmente cette complexité et exige des approches numériques plus complètes. Une phase caractéristique qui interviendra en microgravité dans les futurs lanceurs spatiaux est analysée ici : la dépressurisation des réservoirs cryogéniques. De par les difficultés pour réaliser des essais à grande échelle incluant une phase de microgravité avec un liquide cryogénique, on valide nos méthodes numériques par des essais au sol. Ces essais ont été réalisés sur le site de la Division des Techniques Avancées d'Air Liquide dans le cadre du programme franco-allemand COMPERE.
\end{abstract}

Mots clés : Réservoir cryogénique / microgravité / simulation numérique / dépressurisation / changement de phase / ébullition

\begin{abstract}
CFD application to depressurization of cryogenic tanks in microgravity. In order to control fluid temperature in cryogenic tanks, it is necessary to handle complex thermodynamics phenomena. For future space launchers, phases containing micro-gravity are responsible for an increased complexity and ask for more complete numerical approaches. One characteristic phase occurring in microgravity in future space launchers is analysed in this paper: depressurisation and liquid movements following gravity perturbation. From the difficulties to perform tests with cryogenic liquid including microgravity in large scale facilities, our numerical methods are validated thanks to test performed on earth. These tests have been performed by the Division des Techniques Avancées - Air Liquide in the framework of the FrenchGerman COMPERE program.
\end{abstract}

Key words: Cryogenic tank / microgravity / CFD / depressurization / phase change / boiling

\section{Introduction}

De nombreux travaux passés ou actuels portent sur l'ébullition comparée dans des conditions de microgravité $(\mu \mathrm{g})$ et de gravité normale $(1 \mathrm{~g})$. Ces travaux sont motivés par les applications spatiales et notamment par le besoin de savoir si l'ébullition peut être utilisée comme moyen de transfert de chaleur efficace dans les véhicules spatiaux [1-5].

Les études fondamentales ou appliquées concernent généralement le transfert thermique à la paroi avec un liquide se trouvant à une température égale ou inférieure à la température de vapeur saturante à la pression considérée.

\footnotetext{
a Auteur correspondant :

jerome.lacapere@airliquide.com
}

À partir de nombreuses expériences réalisées en microgravité et de la réflexion théorique, il a été possible d'identifier un mécanisme dominant au niveau de la paroi chauffée : le processus d'évaporation particulièrement intense dans les micro-couches liquides qui se trouvent aux angles des lignes de contact solide/liquide/vapeur [1]. Ce phénomène ne dépendant pas du champ d'accélération, cela explique pourquoi la courbe d'ébullition de Nukiyama (courbe qui donne la densité de flux de chaleur échangée en fonction de la surchauffe $\Delta T=T_{W}-T_{\text {sat }}$ à la paroi; on trouve plusieurs régimes pour $\Delta W$ croissant : ébullition nucléée, crise d'ébullition, zone de transition, film de vapeur) diffère assez peu - en tout cas moins qu'on a pu le penser - en $\mu \mathrm{g}$ et en $1 \mathrm{~g}[1,6]$.

Néanmoins il subsiste des différences. Mais la situation est suffisamment complexe pour que l'on puisse 
penser avec Straub [1], que « même si on comprenait tout, il n'est pas possible de résoudre des problèmes appliqués de transfert de chaleur en présence d'ébullition par simple intégration numérique des équations de conservation $»$.

Cependant, Kim [4] recommande de « continuer le développement des techniques numériques permettant de traiter correctement les écoulements à deux phases ».

Le présent travail concerne une situation différente, où le liquide se trouve surchauffé par baisse de pression, de sorte que l'ébullition en masse est importante et constitue probablement le phénomène dominant. Il est donc logique d'appliquer directement un code numérique diphasique suffisamment sophistiqué à une installation à échelle réelle, sans trop se préoccuper de ce qui se passe aux parois, quitte à perfectionner la description ensuite si les résultats obtenus ne correspondent pas à ceux des expériences.

Durant les différentes phases de vol, les flux thermiques externes chauffent les liquides cryogéniques à l'intérieur des réservoirs et font évaporer une certaine quantité de liquide. De ce fait, la pression dans le réservoir augmente.

Grâce à la dépressurisation, on peut alors remplir un double objectif : faire baisser la pression dans le réservoir pour maintenir un niveau acceptable d'un point de vue structurel et faire baisser la température dans le liquide. En effet, lorsqu'on dépressurise rapidement un réservoir, la température de saturation du fluide liée directement à la pression dans le réservoir chute rapidement. La température du liquide se retrouve ainsi rapidement supérieure à la température de saturation (liquide surchauffé). Le liquide bout en masse jusqu'à ce que sa température redescende à la valeur de la température de saturation pour la pression finale atteinte. Cette forte ébullition est responsable d'une très forte déformation de la surface libre accompagnée de la création de poches gazeuses dans la phase liquide et de la libération de gouttes liquides dans la phase gazeuse. En microgravité, cette déformation de la surface libre s'accompagne d'une très faible, voire d'une absence de ségrégation entre les phases.

Dans un premier temps, on présente le moyen d'essai utilisé ainsi que quelques résultats expérimentaux.

On présente dans une deuxième partie le modèle numérique utilisé ainsi que les résultats numériques obtenus.

On présente également à la fin de cet article un résultat de calcul de dépressurisation réalisé en microgravité.

\section{Moyens d'essais et résultats expérimentaux}

Les essais se sont déroulés en décembre 2002 sur le site de la Division des Techniques Avancées d'Air Liquide à Sassenage. Ils ont été réalisés dans un cryostat en verre (cf. Fig. 1) avec de l'azote liquide. Le cryostat était instrumenté : sondes capacitives, sondes de température, capteur de pression (cf. Fig. 2).

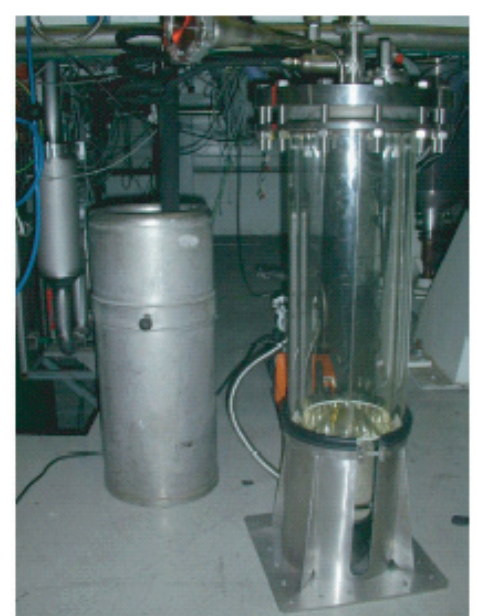

Fig. 1. Photo du cryostat d'essais.

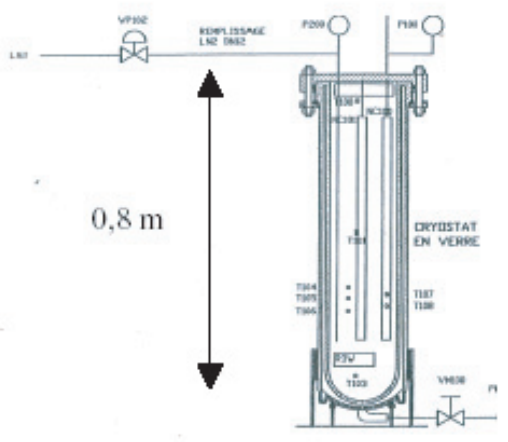

Fig. 2. Représentation schématique du cryostat.

Plusieurs essais de dépressurisation et de ballottement ont été réalisés avec des stratifications thermiques différente dans les phases liquides et gazeuses.

Pendant la première seconde de dépressurisation, aucune bulle n'est présente dans la phase liquide. Le niveau de liquide monte pourtant légèrement. Le régime est établi à partir de 1,4 s. Les bulles se forment, coalescent et sont ensuite libérées dans la phase gazeuse.

La surface libre est déformée mais cette déformation reste légère et il n'y a pas d'expulsions de gouttes liquides dans la phase gazeuse. La dépressurisation représentée à la figure 1 est en fait une dépressurisation de faible intensité (faible pente $\mathrm{d} P / \mathrm{d} t$ ). D'autres dépressurisations plus intenses montrent un phénomène beaucoup plus violent avec détachement de gouttes liquides et une déformation violente de la surface libre.

Les images de la figure 1 ont été prises avec les moyens de visualisations du LEGI (Laboratoire des Écoulements Géophysiques et Industriels de Grenoble).

\section{Modèle et résultats numériques}

De nombreux codes de calculs ont été développés pour la prise en compte du transfert de masse lors d'une 
ébullition. Le but est ici d'utiliser un code généraliste et de l'adapter afin de pouvoir capter ce type de phénomène.

Fluent ${ }^{\circledR}$ est un logiciel utilisé couramment dans l'industrie qui comporte plusieurs modèles multiphasiques dont le modèle VOF (Volume Of Fluid) qui permet la modélisation de plusieurs phases non miscibles. Ce modèle largement répandu est utilisé pour la capture de la surface libre.

Les travaux présentés ici ont été réalisés avec le modèle VOF, le seul capable (parmi ceux proposés par le code Fluent) de déterminer précisément la forme et la position de l'interface liquide-gaz.

Ce modèle, déjà éprouvé a donc été utilisé de manière à bien capter les phénomènes de remontée de bulles, de coalescence, i.e. de surface libre de façon générale.

Les équations génératrices utilisées dans Fluent sont:

$$
\begin{aligned}
& \frac{\partial \varepsilon}{\partial t}+\vec{v} \cdot \nabla \varepsilon=\frac{\dot{m}_{1}}{\rho_{1}} \\
& \frac{\partial}{\partial t}(\rho \vec{v})+\nabla(\rho \vec{v} \cdot \vec{v})= \\
& -\nabla p+\nabla\left[\mu\left(\nabla \vec{v}+\nabla \vec{v}^{T}\right)\right]+\rho \vec{g}+\vec{F} \\
& \frac{\partial}{\partial t}(\rho E)+\nabla \cdot(\vec{v}(\rho E+p))=\nabla \cdot(k \cdot \nabla T)+S_{\mathrm{h}} \\
& \rho=\rho_{1} \varepsilon+\rho_{2}(1-\varepsilon)
\end{aligned}
$$

où $\vec{g}, \rho, \varepsilon, \vec{v}, \mu, E, T, k, \rho_{1}, \rho_{2}$ sont respectivement l'accélération gravitationnelle, la masse volumique, la fraction volumique de la phase 1 , la vitesse, la viscosité dynamique, l'énergie interne massique, la température, la conductivité thermique, la masse volumique de la phase 1 et celle de la phase 2 .

Les propriétés du fluide sont alors calculées suivant les fractions volumiques de chaque phase sur le même type que l'équation (4).

Fluent est un code qui possède des ouvertures par l'intermédiaire des termes sources dans les équations précédentes. Ces termes sources sont à la base des calculs réalisés ici.

Dans le modèle VOF de Fluent 6.0, les termes sources $\dot{m}_{1}$ et $S_{\mathrm{h}}$ sont nuls par défaut.

Le terme source $\vec{F}$ de l'équation (2) inclut la tension de surface par l'intermédiaire du modèle CSF (Continuum Surface Tension) de Brakbill et al. [7]. Ce modèle inclut également une spécification de l'angle statique de contact à la paroi. Les spécifications de la tension superficielle et de l'angle de contact deviennent utiles pour les calculs avec une gravité réduite (faible nombre de Bond).

Dans les modélisations présentées ici, le changement de phase est assuré par les termes sources sur les équations de continuité (1) ainsi que par un terme source sur l'équation de l'énergie (3). Ces termes sources sont calculés par une méthode basée sur l'enthalpie. Cette méthode a souvent fait l'objet de développements notamment en complément de la méthode VOF par Welch et al. [8]. L'intérêt est ici de compléter le logiciel Fluent par cette méthode. En plus des potentialités intrinsèques du modèle VOF de Fluent V6.0 (phase liquide dilatable, phase gazeuse compressible, prise en compte de la tension de surface, transfert thermique entre les phases), on ajoute ainsi le transfert massique entre les phases.

Ainsi, les termes sources des équations (1) et (3) prennent des valeurs différentes suivant la phase :

1. Dans la phase liquide ou phase 1 :

Si la température du liquide devient supérieure à la température de saturation pour la pression considérée, alors les termes sources prennent les valeurs suivantes:

$$
\begin{aligned}
\dot{m}_{2} & =\frac{\varepsilon \cdot \rho_{1} \cdot C_{\mathrm{p}} \cdot\left(T-T_{\mathrm{sat}}\right)}{\Delta t \cdot L} \\
S_{\mathrm{h}} & =-\dot{m}_{2} \cdot L \\
\dot{m}_{1} & =-\dot{m}_{2}
\end{aligned}
$$

\section{Dans la phase gazeuse ou phase 2 :}

Si la température du gaz devient inférieure à la température de saturation pour la pression considérée, alors les termes sources deviennent :

$$
\begin{aligned}
\dot{m}_{2} & =-\frac{(1-\varepsilon) \cdot \rho_{2} \cdot C_{\mathrm{p}} \cdot\left(T-T_{\mathrm{sat}}\right)}{\Delta t \cdot L} \\
S_{\mathrm{h}} & =\dot{m}_{1} \cdot L \\
\dot{m}_{2} & =-\dot{m}_{1}
\end{aligned}
$$

où la capacité calorifique $C_{\mathrm{p}}$ dépend de la température et où la température de saturation $T_{\text {sat }}$ et la chaleur latente $L$ dépendent de la pression (relations de Clausius-Clapeyron), et où $\Delta t$ est la valeur du pas de temps [s] utilisé dans les calculs instationnaires.

La solution utilisée par Voller et al. [9] qui consiste à calculer les termes sources $\dot{m}_{1}$ et $\dot{m}_{2}$ directement à partir du flux thermique échangé entre chaque phase n'est pas applicable ici. En effet, même si les échanges thermiques entre les phases sont loin d'être négligeables dans notre cas, ils ne constituent pas le facteur déclenchant de l'ébullition en masse.

Les relations utilisées ici ont donc l'avantage d'être très souples d'utilisation et de convenir à beaucoup de situations physiques dont l'ébullition nucléée en paroi, la condensation et l'évaporation en surface, ainsi qu'à l'ébullition en masse suite à une dépressurisation.

La figure 4 montre des visualisations de la fraction volumique $\varepsilon$ de liquide dans le cryostat pendant $1,4 \mathrm{~s}$ du début de la dépressurisation. Les conditions de la dépressurisation sont identiques à celles de la dépressurisation expérimentale explicitée au paragraphe précédent.

En bleu, les zones gazeuses où $\varepsilon=0$ et en rouge, les zones liquides où $\varepsilon=1$.

À partir de $t=1,3 \mathrm{~s}$, l'ébullition est en régime établi. Le niveau ne monte plus, les bulles de gaz remontent alors à la surface.

Lors de cette dépressurisation, les informations importantes sont la déformation de la surface libre, la formation des bulles de gaz dans le liquide ainsi que l'évolution de la température du fluide. Qualitativement, la déformation 


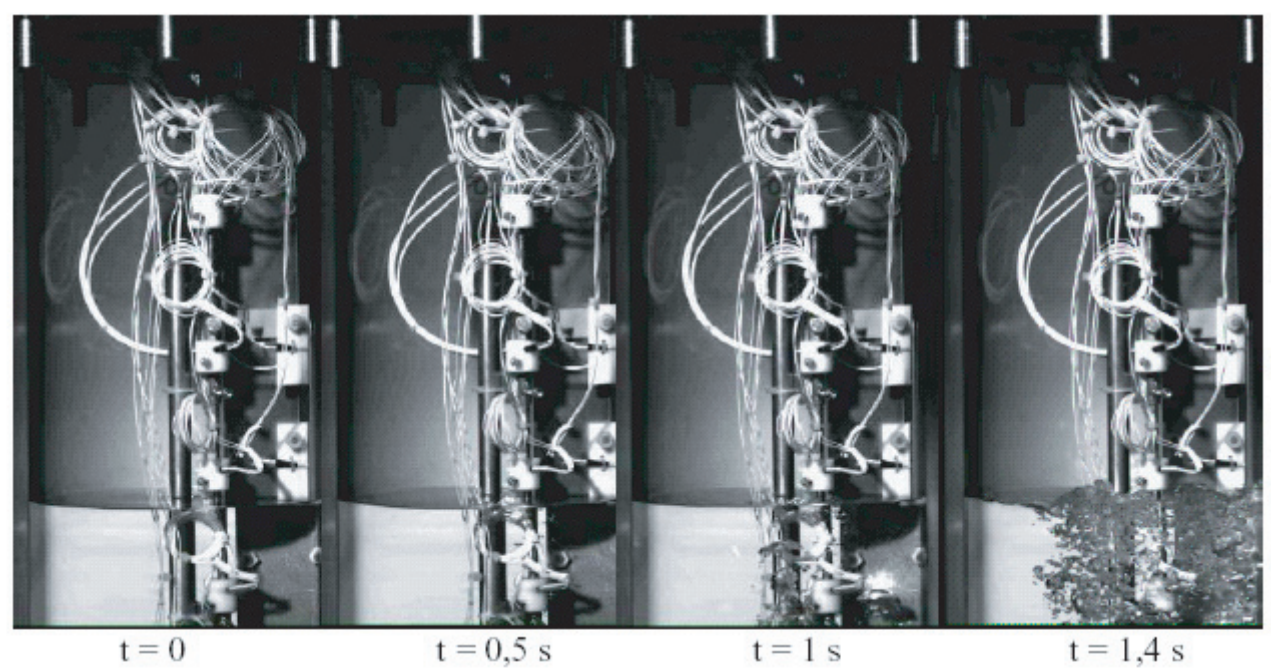

Fig. 3. Visualisation du début de la dépressurisation expérimentale.
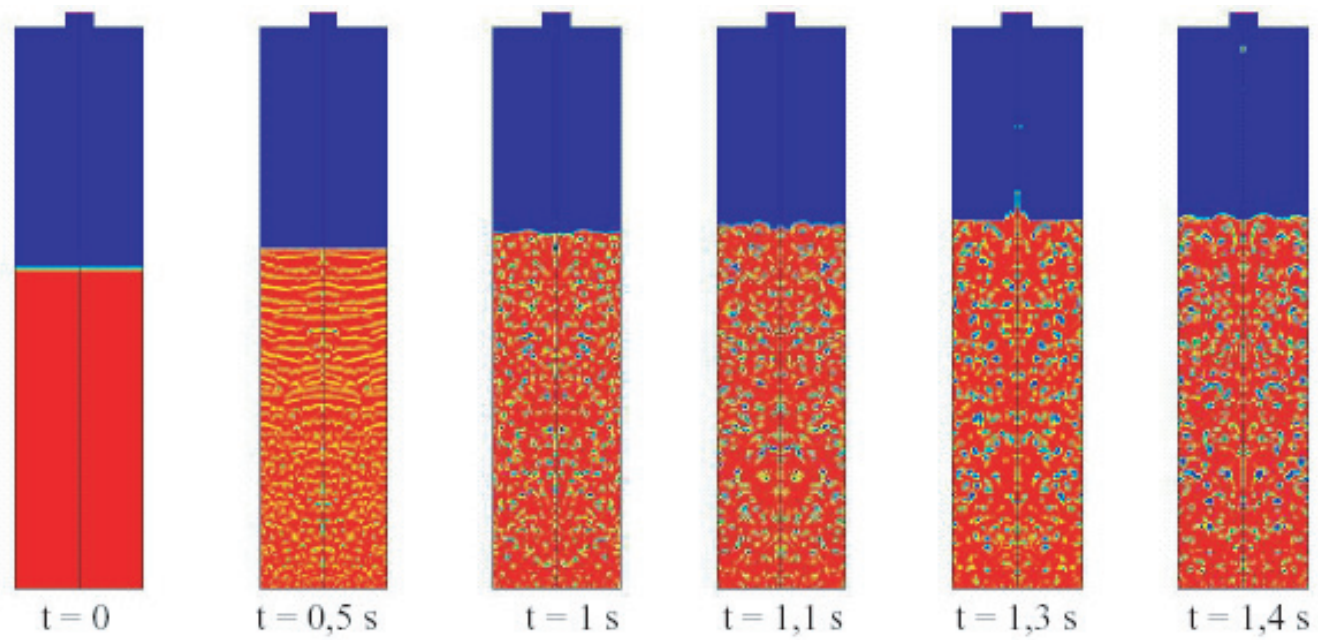

Fig. 4. Représentation des fractions volumiques obtenues par calcul numérique au début de la dépressurisation avec une gravité terrestre $\left(g=9,81 \mathrm{~m} \cdot \mathrm{s}^{-2}\right)$.

de la surface libre et les bulles de gaz générées par Fluent ont un aspect similaire avec la déformation et les bulles de gaz générées expérimentalement. L'élévation du niveau de liquide est cependant légèrement plus forte dans le calcul numérique. On peut attribuer cette différence à notre modélisation en 2D axisymétrique. En effet, dans cette modélisation, les poches gazeuses qui se forment ont un aspect torique, et sont donc plus propices à créer une élévation de niveau qu'une bulle d'aspect plus sphérique que l'on pourrait obtenir en 3D.

La validation de ces méthodes numériques est encore en cours mais on peut d'ores et déjà se servir de ces moyens numériques pour avoir une idée de la déformation de la surface libre lorsqu'on se trouve en microgravité.

Voici donc sur la figure 5 les visualisations de ce qui pourrait se passer lors du début de la même dépressurisation pour une gravité de $10^{-3} \mathrm{~m} . \mathrm{s}^{-2}$.

Les bulles se forment moins rapidement en l'absence de gravité. La ségrégation des phases est moins présente.
Contrairement au cas de la dépressurisation sous une gravité terrestre normale (Fig. 4), on n'assiste pas à la remontée de bulles qui coalescent et à la libération de ces bulles en surface libre. On assiste plutôt ici à une expansion des poches gazeuses entraînant le liquide dans la totalité du domaine. Ainsi, en sortie du domaine, le fluide est composé de liquide et de gaz à partir du temps $t=2,7 \mathrm{~s}$. Ce qui constitue un point critique dans le cadre de nos applications.

\section{Conclusion}

On a présenté ici les résultats d'essais expérimentaux d'une dépressurisation (effectuée au sol) d'un cryostat rempli de LN2. On a également présenté la méthode utilisée pour modéliser cette dépressurisation par calculs numériques. La validation au sol de tels calculs est primordiale pour pouvoir ensuite utiliser le calcul 


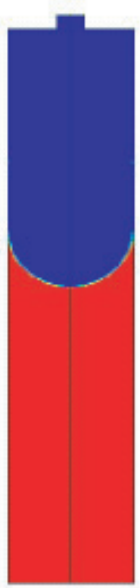

$\mathrm{t}=0$

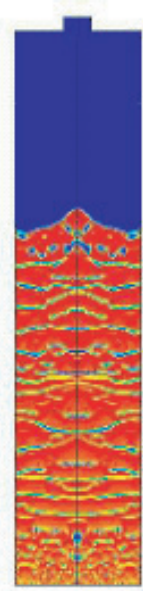

$\mathrm{t}=1 \mathrm{~s}$

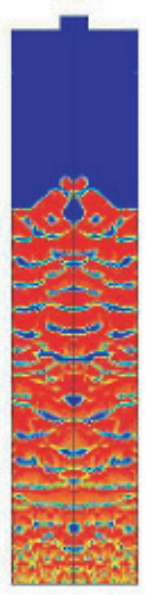

$\mathrm{t}=1,3 \mathrm{~s}$

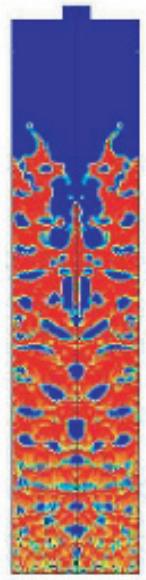

$\mathrm{t}=1,6 \mathrm{~s}$

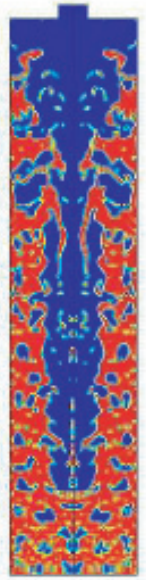

$\mathrm{t}=2,3 \mathrm{~s}$

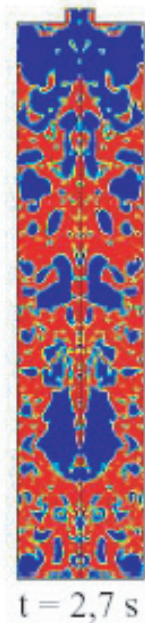

Fig. 5. Représentation des fractions volumiques obtenues par calcul numérique au début de la dépressurisation avec une gravité réduite $\left(g=10^{-3} \mathrm{~m} \cdot \mathrm{s}^{-2}\right)$.

numérique pour des gravités réduites. En effet, l'utilisation de moyens d'essais en microgravité avec des fluides cryogéniques à grande échelle demeure aujourd'hui une tache difficile à réaliser.

Qualitativement, les essais de dépressurisations montrent que la méthode numérique employée donne des résultats satisfaisant.

Nous tenons à remercier le LEGI (Grenoble) pour la qualité des prises de vue fournies durant les différents essais de dépressurisation.

\section{Références}

[1] J. Straub, Boiling Heat Transfer and Bubble Dynamics in Microgravity: Advances in Heat Transfer 35 (2001)

[2] P. Di Marco, Review of Reduced Gravity Boiling Heat Transfer: European Research. J. Jpn. Soc. Microgravity Appl. 20(4) (2003) 252-263
[3] H. Otha, Review of Reduced Gravity Boiling Heat Transfer: Japanese Research. J. Jpn. Soc. Microgravity Appl. 20(4) (2003) 272-285

[4] J. Kim, Review of Reduced Gravity Boiling Heat Transfer: US Research. J. Jpn. Soc. Microgravity Appl. 20(4) (2003) 264-271

[5] P. Arlabosse, C. Reynard, L. Tadrist, Overview of Pool Boiling Heat Transfer studies in Variable Gravity, Space Technology and Applications International Forum, Albuquerque, STAIF, 2000

[6] Jr.H. Merte, J.A. Clark, Boiling heat transfer with cryogenic fluids at standard, fractionnal, and near-zero gravity, J. Heat Transfer 86 (1964) 351

[7] J.U. Brackbill, D.B. Kothe, C. Zemach, A Continuum Method for Modelling Surface Tension, J. Comput. Phys. 100 (1992) 335-354

[8] S.W.J. Welch, J. Wilson, A volume of fluid based method for fluid flows with phase change, J. Comput. Phys. 160 (2000) 662-682

[9] V.R. Voller, M. Cross, C. Markatos, An enthalpy method for convection/diffusion phase change, International Journal For Numerical Methods in Engineering 24 (1987) 271-284 